\title{
Detecting Ischemic Stress to the Myocardium Using Laplacian Eigenmaps and Changes to Conduction Velocity
}

\author{
Wilson W. Good ${ }^{1}$, Burak Erem² ${ }^{2}$ Jaume Coll-Font ${ }^{3}$, Dana H. Brooks ${ }^{3}$, Rob S. MacLeod ${ }^{1}$ \\ ${ }^{1}$ Scientific Computing and Imaging Institute, Bioengineering, University of Utah, Salt Lake City, UT, \\ USA \\ ${ }^{2}$ Boston Children's Hospital and TrueMotion, Boston, MA, USA \\ ${ }^{3}$ SPIRAL Group, ECE Dept., Northeastern University, Boston, MA, USA
}

\begin{abstract}
The underlying pathophysiology of ischemia and its electrocardiographic consequences are poorly understood, resulting in unreliable diagnosis of this disease. This limited knowledge of underlying mechanisms suggests a data driven approach, which seeks to identify patterns in the ECG that can be linked statistically to underlying behavior and conditions of ischemic stress. The gold standard ECG metrics for evaluating ischemia monitor vertical deflections within the ST segment. However, ischemia influences all portions of the electrogram. Another metric that targets the QRS complex during ischemia is Conduction Velocity $(\mathrm{CV})$. An even more inclusive, data driven approach is known as "Laplacian Eigenmaps" (LE), which can identify trajectories, or "manifolds", that respond to different spatiotemporal consequences of ischemic stress, and these changes to the trajectories on the manifold may serve as a clinically relevant biomarker. On this study, we compared the LE- and CV-based markers against two gold standards for detecting ischemic stress, both derived from the ST segment. We evaluated the response time and fidelity of each biomarker using a Time to Threshold (TTT) and Contrast Ratio (CR) measure, over 51 episodes recorded as cardiac electrograms from a canine model of controlled ischemia. The results show that metrics designed to monitor regions beyond the ST segment can perform at least as well, if not better, than traditional ST segment based metrics.
\end{abstract}

\section{Introduction}

The motivation for this research is the persistently poor performance of ECG based methods in diagnosing acute ischemia in the settings of stress testing and the emergency department [1]. Diagnosis based on ECG has both a long history and a compelling rationale; ischemia generates changes in cardiac electrical behavior that should be visible in the ECG. However, identification of robust markers of ischemia remains challenging. Traditional biomarkers of ischemia, such as upward or downward shifts in the ST segment, are thought to be driven by the differences in transmembrane potentials between healthy and ischemic regions. This potential difference gives rise to injury currents which drive diagnostic ST-segment shifts. However, ischemia affects more than the plateau of the action potential, suggesting that additional metrics could reveal ischemic stress earlier and with a greater robustness than current clinical standards. [2] A clear candidate for such a marker is the spread of excitation, which our experimental preparation allowed us to estimate in three dimensions via transmural multielectrode needles. The resulting three-dimensional (3D) view allowed conduction velocity $(\mathrm{CV})$ to be estimated throughout the region sampled by the electrodes. However, conduction velocity only captures the spread of activation in the QRS complex. An even broader approach is to evaluate the entire QRST and for this we employed a machine learning technique known as Laplacian Eigenmaps (LE)[3]. LE is an algorithm that utilizes the entire QRST over all available leads nonlinearly projected onto a lower dimensional parameter space from which differentiating features can be extracted. We applied LE to quantify changes in this lower dimensional space, or LE space, to produce what we call the LE metric. We then evaluated the $\mathrm{CV}$ - and LE-metrics against two ST-based metrics to quantify their performance in detecting acute myocardial ischemia.

\section{Methods}

\subsection{Experimental data collection}

The cardiac electrograms used for this study were collected over 15 in situ canine experiments during which we induced 51 episodes of acute myocardial ischemia. Each ischemic episode, or intervention, lasted 8 minutes with 
a 30-minute downtime to allow the tissue to recover before subsequent episodes. Anywhere from 3-8 interventions were performed per animal, using ischemic protocols designed to produce a graded increase in either supply- or demand-based ischemia in the tissue perfused by the left anterior descending (LAD) artery. Supply-based ischemia was produced by maintaining an elevated heart rate and incrementally reducing blood flow in the LAD over the course of the intervention. Demand-based ischemia was produced by holding a diminished flow rate in the LAD constant while the heart rate was increased. The electrocardiographic response to ischemia was measured using 25-40 transmural plunge needles, each consisting of 10 unipolar electrodes.

\subsection{ST-derived Metrics}

To measure ST-segment shifts, we used the ST40\% potential averaged across all samples in a $10 \mathrm{~ms}$ window centered at the time instant $40 \%$ of the duration from the end of the QRS to the peak of the T wave. This computation was repeated for each electrode across every run in the experiment. For a more detailed analysis of the ST40\% metric, see Aras et al. [4] We compared these results to those based on the ST40 shift, measured at the time instant $40 \mathrm{~ms}$ after the end of the QRS, averaged across a 10-millisecond window.

\subsection{D Conduction Velocity}

3D conduction velocity ( $\mathrm{CV}$ ) estimation was performed throughout the region of tissue sampled by the plunge needles for each run over the course of each experiment. A tetrahedral mesh was created based on the electrode locations as the nodes using Delaunay criteria implemented in the custom software SCIRun (SCI Institute). Any tetrahedra with poor aspect ratio was removed from the mesh. For each face of each tetrahedral element, the CV was estimated using the edge lengths, differences in activation times, and the angles that separate the edges by extending to three dimensions the triangulation technique outlined in Cantwell et al.. [5] The algorithm assumed a planar wave moving through the face and the resulting $\mathrm{CV}$ vector was averaged across the four faces to produce a single representative vector for the entire tetrahedron. We then defined a "CV metric" as the difference between the CV measured during rest and the $\mathrm{CV}$ during the ischemic intervention, a metric of the change in conduction velocity over each run in the experiment.

\subsection{Laplacian Eigenmaps}

Laplacian Eigenmaps (LE) is a dimensionality reduction method that is capable of reducing many simultaneous time signals into a trajectory on a manifold of lower dimensionality. In our implementation, as described in detail in Erem et al.[3], each time point measured across the entire set of electrodes corresponds to a single location on the manifold. These trajectories were obtained by computing a matrix of inverse exponentials of pairwise Euclidean distances between all input points, and then taking its singular value decomposition (SVD). The inverse exponentials are scaled to emphasize local relationships in the data. The SVD determined and ranks the significance of the coordinates in the lower dimensional space. We defined three relevant coordinates to be the second through fourth columns of the right singular vector matrix (the first column was ignored because it is constant.) The manifold coordinates were learned using the beats recorded during the initial rest period before the first intervention was induced. Once the coordinate space was identified, it was populated with subsequent beats over repeated episodes of induced ischemia, as seen in Fig. 1B. [6] [3]
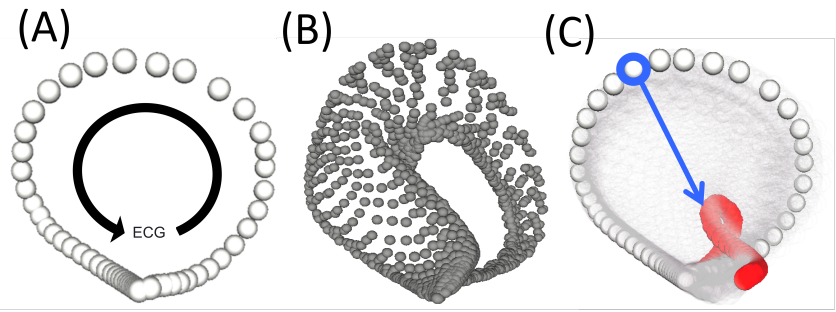

Figure 1. Laplacian Eigenmap manifolds. A.) This manifold consists of a single trajectory corresponding to a run during the rest period, before the induction of ischemia. The progression of the ECG and its relation to the trajectory are shown by the black arrow. B.) This manifold shows several runs mapped into the same manifold space. Each trajectory corresponds to a single run during the intervention. C.) This final manifold shows the trajectory of the run taken at rest (white) and the final run of the intervention (red) with all other trajectories set to be transparent. The blue arrow corresponded to the point on the trajectory that showed the greatest sensitivity to the underlying ischemic stress.

The manifold produced by this analysis consisted of a single trajectory for every run in the experiment. As progressively ischemic runs were mapped into the manifold space, large regions of the trajectories responded to the underlying stress state. To determine which point on the trajectory responded most robustly, we measured the Euclidean distance between each point on the healthy trajectory and its corresponding point on each subsequent trajectory for each run in the experiment. The trajectory point that showed the greatest CR was chosen as the LE metric for that intervention. The magnitude of movement along 
the blue arrow, seen in Fig. 1C, was used as the LE metric. [7]

\subsection{Measures of Metric Quality}

Two measures were developed to quantify how early each metric was able to detect ischemia and the robustness with which each metric responded. These metrics are calculated on the representative beats we extracted over the course of the experiments. We will refer to each extracted beat as a 'run", defined here as the first representative beat per continuous recording (typically $3 \mathrm{~s}$ in duration taken every 15 seconds during the ischemic interventions and every ten minutes during the rest periods). We captured 80-300 such runs over the course of each experiment. To follow the progression of metrics over the experiments, we defined "run-metric plots", continuous plots of each metric over one or more intentions (see Figure 2). We also defined the Time to Threshold (TTT) as a measure of how early each metric responds and the Contrast Ratio (CR) as the magnitude of the response with respect to the mean amplitude at rest extracted from the run-metric plots.

$$
\begin{aligned}
& \text { TTT }=\overline{A_{r}}+\frac{A_{m}-\overline{A_{r}}}{3} \\
& \text { Contrast Ratio }(\mathbf{C R}):=\frac{A_{m}}{\overline{A_{r}}},
\end{aligned}
$$

where $\overline{A_{r}}$ is the mean value at rest or control of any metric and $A_{m}$ is the maximum value of the metric.

\section{Results}

\subsection{Time to Threshold and Contrast Ratio}
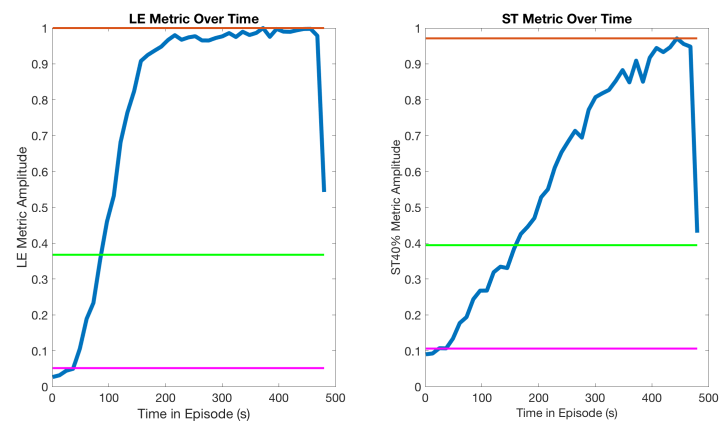

Figure 2. LE and ST40\% run-metric plots for the same ischemic episode. Each metric has been normalized to put the metrics on a common scale. A.) The LE metric over time. B.) The ST40\% metric over time. Magenta line: Average value at rest $\left(\overline{A_{r}}\right)$. Orange line: Maximal value during intervention $\left(A_{m}\right)$. Green line: Automatically determined threshold.
Table 1 shows the values of TTT and CR for all the metrics we compared. Of the 51 interventions evaluated, the LE metrics detected ischemia earlier than the ST $40 \%$ metric in 42 of the episodes with a mean decrease in detection time of $36.4 \mathrm{~s}$. The ST40\% metric detected ischemia earlier than the CV metric in 38 of the episodes with an average difference of $32.7 \mathrm{~s}$. A comparison of the two traditional metrics showed no meaningful differences in performance. The results for CR, showed that both $\mathrm{LE}$ and $\mathrm{CV}$ metrics exhibited a greater degree of contrast from baseline levels ( 5.5 and 5.9 for LE and CV, respectively) than the two ST segment based metrics (3.5 and 3.6 for ST40 and ST40\%).

Table 1. Evaluating the TTT and CR of Four Metrics Designed to Detect Ischemic Stress

\begin{tabular}{lcc}
\hline \hline Metric & TTT (s) & CR \\
\hline ST40\% & 262.8 & 3.6 \\
ST40 & 264.2 & 3.5 \\
CV & 290.5 & 5.9 \\
LE & 226.5 & 5.5 \\
\hline \hline
\end{tabular}

\subsection{Conduction Velocity During Acute Is- chemia}

While the purpose of this study was to evaluate how various metrics compared in detecting ischemic stress, the computation of $\mathrm{CV}$ throughout the transmural extent also allowed us to measure the influence of ischemia on conduction velocity. The proarrhythmic vulnerability induced in ischemic tissue is well known but poorly understood and has yet to be measured in three dimensions throughout even a reasonably sized region of the myocardium. As seen in Table 2, the healthy CVs of $61 \mathrm{~cm} / \mathrm{s}$ and ischemic CVs of $47 \mathrm{~cm} / \mathrm{s}$ were comparable to the one- and two-dimensional measures reported in the literature. [2] The framework for measuring $\mathrm{CV}$ proposed here will be utilized in future studies to investigate the vulnerabilities created by acute myocardial ischemia.

Table 2. Conduction Velocity During Acute Myocardial Ischemia

\begin{tabular}{lc}
\hline \hline Tissue State & Conduction Velocity $(\mathrm{cm} / \mathrm{s})$ \\
\hline Healthy & $61.1 \pm 12$ \\
Ischemic & $46.6 \pm 11$ \\
\hline \hline
\end{tabular}

\section{Discussion and Conclusions}

Traditional means of detecting and diagnosing ischemia have relied on amplitude shifts within the ST segment, 
which are not unique to ischemia. The goal of this research was to identify metrics capable of robustly detecting ischemia with a short response time. For this end, we first developed the CV metric designed to use the 3D architecture provided by our transmural needles to estimate the activation wavefront as it moves through the myocardium. Clearly this metric can only be calculated when transmural measurements are available. In addition we applied the LE metric as a data driven approach that does not presume a bias based on any mechanisms. This approach is also attractive as it has the potential to maintain sensitivity to ischemia as one moves from the needles to the epicardium and the torso surface. We found that while the LE metric was less robust than the $\mathrm{CV}$ metric, it was the earliest responding metric of those considered. Furthermore, we found that the heart rate adjusting metric, ST40\%, performed similarly to the metric that did not account for heart rate variability, ST40. The similarity of the ST-metrics may suggest that adjusting for heart rate may be unnecessary for earlier detection of ischemia. The ST-metrics had lower CRs than both the LE- and CV-metrics, suggesting regions beyond the ST segment could be used in conjunction with traditional metrics to detect ischemia with greater robustness. The early and robust detection of ischemic stress by the LE metric prompts further study into its performance on epicardial and torso potentials to assess its clinical viability.

The CV metric leverages the changes seen in the spread of activation, as measured by the activation time of the extracellular electrogram, which corresponds well to the upstroke of the action potential. While we could detect changes in activation, the $\mathrm{CV}$ metric we derived did not respond as quickly to ischemic stress as ST-segment based metrics. The ability to measure the development of ischemia in 3D and monitor how it influences the CV of the surrounding myocardium provides a platform for future studies. The reasons for the earlier response of the LE metric is unclear as the physiological basis driving the changes seen in the LE space have not yet been clearly identified. The fact that the entire signal is used to learn the LE manifold coordinates may suggest its performance is due to its ability to be sensitive to any and all changes seen in the electrograms.

\section{Acknowledgements}

Support for this research comes from the NIH NIGMS Center for Integrative Biomedical Computing (www.sci.utah.edu/cibc), NIH NIGMS grant no. P41GM103545, the Nora Eccles Treadwell Foundation for Cardiovascular Research.

\section{References}

[1] Stern S. State of the art in stress testing and ischaemia monitoring. Cardiac electrophysiology review 2002;6(3):204208.

[2] Kleber AG, Janse MJ, Wilms-Schopmann F, Wilde A, Coronel $\mathrm{R}$. Changes in conduction velocity during acute ischemia in ventricular myocardium of the isolated porcine heart. Circulation 1986;73(1):189-198.

[3] Erem B, Orellana RM, Hyde DE, Peters JM, Duffy FH, Stovicek P, Warfield SK, MacLeod RS, Tadmor G, Brooks DH. Extensions to a manifold learning framework for timeseries analysis on dynamic manifolds in bioelectric signals. Physical Review E 2016;93(4):042218.

[4] Aras K, Burton B, Swenson D, MacLeod R. Sensitivity of epicardial electrical markers to acute ischemia detection. Journal of electrocardiology 2014;47(6):836-841.

[5] Cantwell CD, Roney CH, Ng FS, Siggers JH, Sherwin S, Peters NS. Techniques for automated local activation time annotation and conduction velocity estimation in cardiac mapping. Computers in biology and medicine 2015;65:229-242.

[6] Belkin M, Niyogi P. Laplacian eigenmaps for dimensionality reduction and data representation. Neural computation 2003; 15(6):1373-1396.

[7] Good WW, Erem B, Coll-Font J, Brooks DH, MacLeod RS. Novel biomarker for evaluating ischemic stress using an electrogram derived phase space. Computing in Cardiology Conference CinC 2016 2016;1057-1060.

Address for correspondence:

Name: Wilson William Good

Full postal address: SCI Institute, University of Utah, 72 Central Campus Dr, Salt Lake City, UT 84112

E-mail address: wgood@sci.utah.edu 